\title{
A fuzzy inventory model with imperfect items and backorder with allowable proportionate discount
}

\author{
Rojalini Patro ${ }^{1}$, Sujit Acharya ${ }^{2}$, Mitali Madhusmita Nayak ${ }^{1 *}$, Milu Acharya ${ }^{1}$ \\ ${ }^{1}$ Department of Mathematics, Siksha 'O' Anusandhan Deemed to be University, Bhubaneswar-751030, Odisha, India \\ ${ }^{2}$ Department of Management Studies, DDCE, Utkal University, Bhubaneswar - 751007, Odisha, India
}

Corresponding Author Email: mitalinayak7@ gmail.com

https://doi.org/10.18280/mmc_d.390106

Received: 23 January 2018

Accepted: 20 December 2018

\section{Keywords:}

inventory, imperfect quality, proportionate discount, backorder, EOQ

\begin{abstract}
This paper presents both crisp and fuzzy EOQ models for defective items present in each lot when shortages are allowed and backorder takes place. The aim of the work is to first construct an optimal order quantity for the crisp case and then to develop the corresponding fuzzy model. In contrast to the previous inventory models, an allowable proportionate discount is incorporated for the defective items present in each lot to provide a general framework to the model. The aim of the present paper is to find the optimal order size and the expected shortage level so as to obtain the optimum total profit for both the models. The necessary and sufficient conditions for the existence and uniqueness of the optimal solutions are derived and it is also shown that under certain conditions the crisp model boils down the traditional EOQ backorder formula. For the fuzzy case, triangular fuzzy numbers are used for the defective rates and for defuzzification signed distance method is used. Finally, numerical example is provided to illustrate the solution procedure and sensitivity analysis is performed on the results to analyze the effect of the variations taken place for the parameters involved in the model.
\end{abstract}

\section{INTRODUCTION}

Harris's [7] is the first who presented the traditional EOQ formula which appears in almost all text books covering inventory management. Thereafter, many researchers have extensively analysed on economic lot size problems by changing some of the original assumptions of the traditional EOQ model. The traditional EOQ model includes few unrealistic assumptions. One of the unrealistic assumptions which the traditional EOQ model holds is the received quantities are all of perfect qualities. But practically, this assumption is very impractical. To address this many authors have developed various EOQ models considering the lot with imperfect type items. Porteus [17] was the first researcher who gave attention on imperfect quality items. He incorporated the effect of defective items into the traditional EOQ model. At the same time Rosenblatt and Lee [12] focused on rework of defective items and found that the presence of defective products causes smaller lot sizes. The assumption of imperfect quality was first initiated by Salameh and Jaber [20] and they assumed that the defective items could be sold at a discounted price in a single batch at the end of $100 \%$ screening of all the received items. Goyal and Cardnas-Baron [6] extended the work of Salameh and Jaber [20] and they developed a practical approach to optimize the lot size. Wahab and Jaber [22] extended the models of Salameh and Jaber [20] and Maddah and Jaber [14] and presented an optimal lot size for items with imperfect quality under the consideration of two types of holding costs. Many researchers have extended the work of the original model proposed by Salameh and Jaber [20] in between 2000 - 2015. But, Rezaei [18] deed the extension of an EOQ model with shortages backordered. He was the first person who brought the concept of shortages in the model of Salameh and Jaber [20]. He introduced the concept of cyclic shortage resulting out of the imperfect production, leads to backorder in the beginning of each cycle. Wee et al. [23] developed an inventory model with items of imperfect quality and shortages are backordered by allowing $100 \%$ screening of items where, the screening rate is greater than the demand rate. Subsequently, Eroglu and Ozdemir [5] established an inventory model with some percentage of defectives by allowing shortages to be fully backordered. Chang \& Ho [2] provided closed-form solutions using the renewal reward theorem. However, in their work they assumed that the items satisfying the backorder are delivered without any screening process. Hsu and Hsu [8] considered the inventory model with multiple screening for items of imperfect quality and shortages backordered. Jagadeeswari and Chenniappan [9] extended an inventory model by taking quadratic time dependent demand for deteriorating items with shortages are partially backlogged. Khanna et al. [10] developed an inventory model for items of imperfect quality with deterioration under trade-credit policies having selling price dependent demand and shortages which are fully backlogged. The traditional inventory models including uncertainties and randomness were handled with the help of probability theory. Specifically, when there is a lack of historical data to estimate the probability distributions for different uncertain factors present in the model, we prefer to include fuzzy numbers. Thus, considering the inventory model of Salameh and Jaber [20], researchers like Chang [1], Chen and Chang [3] developed inventory models by introducing fuzzy numbers for different parameters involved in it. Lee and Yao [13] developed an economic production quantity (EPQ) model in which the demand and the 
production quantity are assumed to be fuzzy. De and Rawat [4] developed a fuzzy inventory model without shortages by a using triangular fuzzy numbers. Kumar and Goswami [11] developed a fuzzy EOQ inventory model for items with imperfect quality where shortages are completely backlogged. Sujatha and Parvathi [21] investigated an inventory model for deteriorating items with shortages under partially backlogging where the demand rate is two parameter Weibull distribution and the inventory costs are taken to be triangular fuzzy numbers. Recently, Patro et al. [16] established both crisp and fuzzy Economic order quantity (EOQ) models with proportionate discount for items with imperfect quality under learning effect over a finite time horizon. Patro et al. [15] have also developed a fuzzy inventory model for time dependent Weibull deterioration and quadratic demand rate where shortages are allowed and are partially backlogged.

In this paper, we develop a fuzzy EOQ inventory model of imperfect quality items, present in each lot, with shortages by introducing allowable proportionate discount. To obtain the total profit, a $100 \%$ screening is conducted for the lot and an allowable proportionate discount is introduced for the defective items. In case of the fuzzy inventory model, the defective rates are taken to be fuzzy with the allowable proportionate discount for the defective items present in each lot, where the total profit per unit time is derived in fuzzy sense. The fuzzy EOQ models are derived by using the triangular fuzzy numbers for the defective rates and the fuzzy models are defuzzified by using the signed distance method. The solutions for maximizing the fuzzy total profits per unit time have been derived and numerical examples are given to illustrate the model. Finally, the sensitivity analysis is performed on the results to analyse the effect of the variations taken for the parameters involved in the model.

\section{THE MATHEMATICAL MODEL}

\subsection{Crisp model}

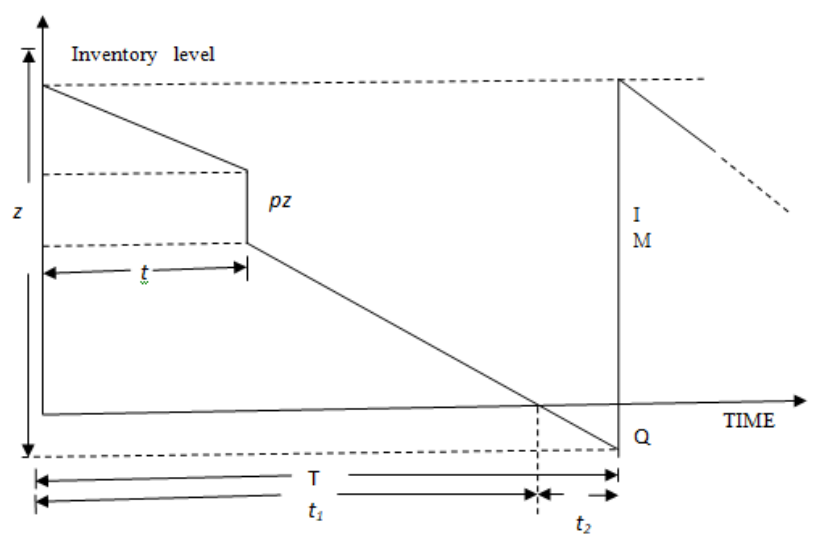

Figure 1. Behaviour of the inventory level over time

Using the above assumption, the cycle wise total cost is given as follows:

$f(z, Q)=$ Ordering cost + unit cost of variable + screening cost with regard to cycle wise lot

size + holding cost + Shortage cost $f(z, Q)=C_{k}+C_{p} z+C_{s} z+C_{h} \times\left(\frac{z(1-p)-Q}{2} t_{1}+\frac{p z^{2}}{w}\right)+$

$\frac{Q C_{b}}{2} t_{2}$

The cycle wise total revenue is given as follows:

$g(z, Q)=$ total sales with regard to good quality items + total sales with regard to imperfect type of items.

$g(z, Q)=S_{g} z(1-p)+\sum_{i=1}^{z p}\left(S_{g}-\left(1-\frac{z p-i}{z p}\right)\left(\frac{g(z, Q)-f(z, Q)}{z}\right)\right)$

After simplification the result obtained for $g(z, Q)$ is given as follows.

$g(z, Q)=\frac{2 S_{g} z^{2}+\left(C_{k}+C_{p} z+C_{s} z+C_{h} \times\left(\frac{z(1-p)-Q}{2} t_{1}+\frac{p z^{2}}{w}\right)+\frac{Q C_{b}}{2} t_{2}\right)(z p+1)}{2 z+(z p+1)}$

The cycle wise total profit $\pi(z, Q)$ is the difference between the cycle wisetotal revenue and the cycle wise total cost per cycle.

$$
\begin{aligned}
& \pi(z, Q)=g(z, Q)-f(z, Q) \\
& \pi(z, Q)=\frac{2 S_{g} z^{2}+\left(C_{k}+C_{p} z+C_{s} z+C_{h} \times\left(\frac{z(1-p)-Q}{2} t_{1}+\frac{p z^{2}}{w}\right)+\frac{Q C_{b}}{2} t_{2}\right)(z p+1)}{2 z+(z p+1)} \\
& -\left[C_{k}+C_{p} z+C_{s} z+C_{h} \times\left(\frac{z(1-p)-Q}{2} t_{1}+\frac{p z^{2}}{w}\right)+\frac{Q C_{b}}{2} t_{2}\right] \\
& =\frac{2 S_{g} z^{2}-2 z\left(C_{k}+C_{p} z+C_{s} z+C_{h} \times\left(\frac{z(1-p)-Q}{2} t_{1}+\frac{p z^{2}}{w}\right)+\frac{Q C_{b}}{2} t_{2}\right)}{2 z+(z p+1)}
\end{aligned}
$$

The total profit per unit time is denoted by $\pi_{u}(z, Q)$ and the formula used for the total profit is given as follows.

$$
\pi_{u}(z, Q)=\frac{\pi(z, Q)}{T}
$$

where $T=\frac{z(1-p)}{D}$ and replacing $t_{1}$ and $t_{2}$ by $\frac{z(1-p)-Q}{D}$ and $\frac{Q}{D}$ respectively in equation (2.1.3) the total profit per unit time can be written as:

$$
\begin{aligned}
& \pi_{u}(z, Q)=\frac{2 D\left[S_{g} z-C_{k}-C_{p} z-C_{S} z\right]}{(1-p)(2 z+z p+1)}-\left[\frac{C_{h} z}{2 z+z p+1}\{z+p z-2 Q\}+\right. \\
& \left.\frac{Q^{2}\left(C_{b}+C_{h}\right)}{(1-p)(2 z+z p+1)}\right]
\end{aligned}
$$

Since $p$ is the percentage of defective items, which is received with a known probability density functions, then the Eq. (2.1.4). $E \pi_{u}(z, Q)$, is given as follows:

$$
\begin{aligned}
& E \pi_{u}(z, Q)=\frac{2 D\left[S_{g} z-C_{k}-C_{p} z-C_{s} z\right]}{(1-E[p])(2 z+z E[p]+1)} \\
& =-\left[\frac{C_{h} z\{z+E[p] z-2 Q\}}{2 z+z E[p]+1}+\frac{Q^{2}\left(C_{b}+C_{h}\right)}{(1-E[p])(2 z+z E[p]+1)}\right]
\end{aligned}
$$


The optimality condition for the nonlinear problem the expected total profit per unit time given in Eq. (2.1.5), is demonstrated by finding the first and second partial derivatives of $E \pi_{u}(z, Q)$ with respect to $z, Q$ are obtained as follows:

$$
\begin{aligned}
& \frac{\partial E T P U(z, Q)}{\partial z}=\left(\frac{1}{1-E[p]}\right)\left(\frac{1}{(2 z+z E[p]+1)^{2}}\right) \\
& =\left[\begin{array}{l}
2 D S_{g}-2 D C_{p}-2 D C_{s}+4 D C_{k}+2 D C_{k} E[p]-2 C_{h} z^{2}-2 C_{h} z^{2}(E[p])+ \\
3 C_{h} z^{2}(E[p])^{2}-2 C_{h} z+2 Q C_{h}+C_{h} z^{2}(E[p])^{3}+2 C_{h} z(E[p])^{2} \\
-2 Q C_{h} E[p]+2 Q^{2} C_{b}+2 Q^{2} C_{h}+Q^{2} C_{b} E[p]+Q^{2} C_{h} E[p]
\end{array}\right]
\end{aligned}
$$

$$
\frac{\partial E T P U(z, Q)}{\partial Q}=\frac{2 C_{h} z}{(2 z+z E[p]+1)}-\frac{2 Q\left(C_{b}+C_{h}\right)}{(1-E[p])(2 z+z E[p]+1)}
$$

By setting (2.1.6(a)) and (2.1.6(b)) equal to zero, we obtain:

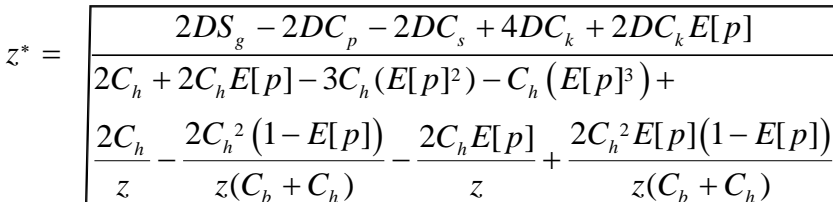

$$
\begin{aligned}
& \mid \begin{array}{l}
-\frac{2 C_{b} C_{h}{ }^{2}(1-E[p])^{2}}{\left(C_{b}+C_{h}\right)^{2}}-\frac{2 C_{h}{ }^{3}(1-E[p])^{2}}{\left(C_{b}+C_{h}\right)^{2}} \\
-\frac{C_{b} C_{h}{ }^{2} E[p](1-E[p])^{2}}{\left(C_{b}+C_{h}\right)^{2}}-\frac{C_{h}{ }^{3} E[p](1-E[p])^{2}}{\left(C_{b}+C_{h}\right)^{2}}
\end{array}
\end{aligned}
$$

For large value of $z, \frac{1}{z} \rightarrow 0$

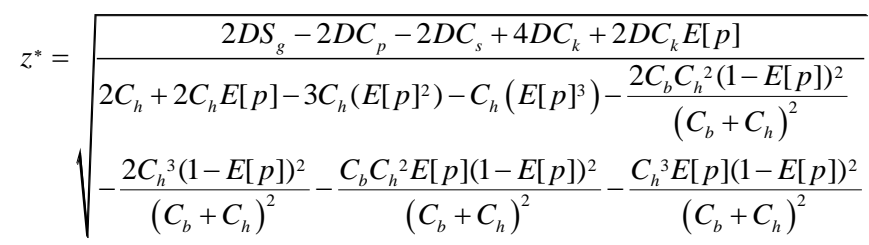

$Q^{*}=\left(\frac{C_{h} z(1-E[p])}{C_{b}+C_{h}}\right)$

$$
I M=z-Q
$$

The maximum inventory level is obtained by substituting the value of Eq.(3.1.7) and Eq.(3.1.8) in equation Eq. (3.1.9) we get the following:

$$
I M^{*}=\frac{z^{*}\left(C_{b}+C_{h} E[p]\right)}{C_{b}+C_{h}}
$$

Now, we obtain the Hessian matrix $\mathrm{H}$ to examine the sufficient conditions for getting maximum value is given as follows:

$$
H=\left[\begin{array}{cc}
\frac{\partial^{2} E \pi_{u}(z, Q)}{\partial z^{2}} & \frac{\partial^{2} E \pi_{u}(z, Q)}{\partial z \partial Q} \\
\frac{\partial^{2} E \pi_{u}(z, Q)}{\partial Q \partial z} & \frac{\partial^{2} E \pi_{u}(z, Q)}{\partial Q^{2}}
\end{array}\right]
$$

$$
\frac{\partial^{2} E \pi_{u}(z, Q)}{\partial z^{2}}=-\left(\frac{1}{1-E[p]}\right)\left(\frac{2}{(2 z+z E[p]+1)^{3}}\right)
$$$$
=\left[\begin{array}{l}
4 D S_{g}-4 D C_{p}-4 D C_{s}+8 D C_{k}+8 D C_{k} E[p]+2 D S_{g} E[p] \\
-2 D C_{p} E[p]-2 D C_{s} E[p]+2 D C_{k}(E[p])^{2}-z C_{h}(E[p])^{2} \\
+C_{h} z E[p]+C_{h}-C_{h}(E[p])^{2}+4 Q C_{h}-2 Q C_{h} E[p] \\
+4 Q^{2} C_{b}+4 Q^{2} C_{h}+4 Q^{2} C_{b} E[p]+4 Q^{2} C_{h} E[p] \\
-2 Q C_{h}(E[p])^{2}+Q^{2} C_{b}(E[p])^{2}+Q^{2} C_{h}(E[p])^{2}
\end{array}\right]
$$

$\frac{\partial^{2} E \pi_{u}(z, Q)}{\partial z \partial Q}=\frac{2 C_{h}-2 C_{h} E[p]+4 Q C_{b}+4 Q C_{h}+2 Q C_{b} E[p]+2 Q C_{h} E[p]}{(1-E[p])(2 z+z E[p]+1)^{2}}$

$\frac{\partial^{2} E \pi_{u}(z, Q)}{\partial Q \partial z}=\frac{2 C_{h}-2 C_{h} E[p]+4 Q C_{b}+4 Q C_{h}+2 Q C_{b} E[p]+2 Q C_{h} E[p]}{(1-E[p])(2 z+z E[p]+1)^{2}}$

$$
\frac{\partial^{2} E \pi_{u}(z, Q)}{\partial Q^{2}}=\frac{-2\left(C_{b}+C_{h}\right)}{(1-E[p])(2 z+z E[p]+1)}
$$

The first principal minor of $\mathrm{H}$ is:

$$
\begin{aligned}
& \left|H_{11}\right|=-\left(\frac{1}{1-E[p]}\right)\left(\frac{2}{(2 z+z E[p]+1)^{3}}\right) \\
& {\left[\begin{array}{l}
4 D S_{g}-4 D C_{p}-4 D C_{s}+8 D C_{k}+8 D C_{k} E[p]+2 D S_{g} E[p] \\
-2 D C_{p} E[p]-2 D C_{s} E[p]+2 D C_{k}(E[p])^{2}-z C_{h}(E[p])^{2} \\
+C_{h} z E[p]+C_{h}-C_{h}(E[p])^{2}+4 Q C_{h}-2 Q C_{h} E[p]+4 Q^{2} C_{b} \\
+4 Q^{2} C_{h}+4 Q^{2} C_{b} E[p]+4 Q^{2} C_{h} E[p]-2 Q C_{h}(E[p])^{2} \\
+Q^{2} C_{b}(E[p])^{2}+Q^{2} C_{h}(E[p])^{2}
\end{array}\right]<0}
\end{aligned}
$$

The second principal minor of $\mathrm{H}$ is:

$$
\begin{aligned}
& \left|H_{22}\right|=\frac{4}{(1-E[p])^{2}(2 z+z E[p]+1)^{4}} \\
& {\left[\begin{array}{l}
4 D S_{g} C_{b}-4 D C_{p} C_{b}-4 D C_{s} C_{b}-8 D C_{k} C_{b}+8 D C_{k} C_{b} E[p] \\
+2 D S_{g} C_{b} E[p]-2 D C_{p} C_{b} E[p]-2 D C_{s} C_{b} E[p]+2 D C_{k} C_{b}(E[p])^{2} \\
-z C_{h} C_{b}(E[p])^{2}+C_{h} C_{b} z(E[p])+C_{h} C_{b}-C_{h} C_{b}(E[p])^{2} \\
+4 D S_{g} C_{h}-4 D C_{p} C_{h}-4 D C_{s} C_{h}+8 D C_{k} C_{h}+8 D C_{k} C_{h}(E[p]) \\
+2 D S_{g} C_{h}(E[p])-2 D C_{p} C_{h}(E[p])-2 D C_{s} C_{h}(E[p]) \\
+2 D C_{k} C_{h}(E[p])^{2}-z C_{h}{ }^{2}(E[p])^{2}+C_{h}{ }^{2} z(E[p]) \\
-2 C_{h}{ }^{2}(E[p])^{2}+2 C_{h}{ }^{2}(E[p])
\end{array}\right]>0}
\end{aligned}
$$

At point $\left(z^{*}, Q^{*}\right)$ the Hessian Matrix $H$ is negative definite. Therefore, there exist the unique values of $z^{*}$ and $Q^{*}$ in (2.1.7) and (2.1.8) respectively that maximizes $E \pi_{u}(z, Q)$. 
When $p=0, C_{s}+C_{p}=S_{g}$, equation (3.1.7) reduces to the traditional EOQ backorder formula

$$
z=\sqrt{\frac{2 C_{k} D}{C_{h}}\left(\frac{C_{b}+C_{h}}{C_{b}}\right)}
$$

\subsection{Fuzzy model}

Total profit per unit time is given by

$\pi(z, B)=\frac{2 D\left(S_{g} z-C_{k}-C_{p} z-C_{s} z\right)}{(1-p)(2 z+z p+1)}-\left[\frac{C_{h} z\{z+p z-2 B\}}{2 z+z p+1}+\frac{B^{2}\left(C_{b}+C_{h}\right)}{(1-p)(2 z+z p+1)}\right]$

Fuzziness of the defective rate $p$ (or equivalently, the fuzziness of good-quality rate; $1-p$ )

Let $q=1-p$ and rewrite the above expression as follows: $\pi(z, B)=\frac{2 D\left(S_{g} z-C_{k}-C_{p} z-C_{s} z\right)}{q(3 z-z q+1)}-\frac{C_{h} z\{2 z-q z-2 B\}}{(3 z-z q+1)}-\frac{B^{2}\left(C_{b}+C_{h}\right)}{q(3 z-z q+1)}$

Let us consider $q=1-p$ as the triangular fuzzy number, $\tilde{q}=\left(q-\Delta_{1}, q, q+\Delta_{2}\right)$, where $0<\Delta_{1}<q$ and $0<\Delta_{2} \leq 1-$ $q$. Then the total profit per unit time can be written in fuzzy sense which is given as follows:

$\tau(z, B)=\frac{2 D\left(S_{g} z-C_{k}-C_{p} z-C_{s} z\right)}{\tilde{q}(3 z-z \tilde{q}+1)}-\frac{C_{h} z\{2 z-\tilde{q} z-2 B\}}{(3 z-z \tilde{q}+1)}-\frac{B^{2}\left(C_{b}+C_{h}\right)}{\tilde{q}(3 z-z \tilde{q}+1)}$

Now, we defuzzify $\pi(z, B)$ using the signed distance method. The signed distance of $\pi(z, B)$ to $\tilde{0}_{1}$ is given as follows.

$$
\begin{aligned}
& d\left(\pi(z, B), \tilde{0}_{1}\right)=\frac{2 D\left(S_{g} z-C_{k}-C_{p} z-C_{s} z\right)}{\left(3 z-z d\left(\tilde{q}, \tilde{0}_{1}\right)+1\right)} d\left(\frac{1}{\tilde{q}}, \tilde{0}_{1}\right)-\frac{C_{h} z\left\{2 z-d\left(\tilde{q}, \tilde{0}_{1}\right) z-2 B\right\}}{\left(3 z-z d\left(\tilde{q}, \tilde{0}_{1}\right)+1\right)} \\
& -\frac{B^{2}\left(C_{b}+C_{h}\right)}{\left(3 z-z d\left(\tilde{q}, \tilde{0}_{1}\right)+1\right)} d\left(\frac{1}{\tilde{q}}, \tilde{0}_{1}\right)
\end{aligned}
$$

The total profit per unit time in fuzzy sense $\pi(\tilde{z}, B)$, given below, is obtained by substituting the results of $d\left(\tilde{q}, \tilde{0}_{1}\right)$ and $d\left(\frac{1}{\tilde{q}}, \tilde{0}_{1}\right)$ in the above equation we get the following:

$$
\begin{aligned}
& \underset{\pi(z, B)}{\sim}=d\left(\tilde{\pi(z, B)} \tilde{0}_{1}\right)=\frac{1}{2}\left(\frac{1}{\Delta_{1}} \ln \frac{q}{q-\Delta_{1}}-\frac{1}{\Delta_{2}} \ln \frac{q}{q+\Delta_{2}}\right) \frac{2 D\left(S_{g} z-C_{k}-C_{p} z-C_{s} z\right)}{\left(3 z-z\left(q+\frac{\Delta_{2}-\Delta_{1}}{4}\right)+1\right)} \\
& -\frac{C_{h} z\left\{2 z-\left(q+\frac{\Delta_{2}-\Delta_{1}}{4}\right) z-2 B\right\}}{\left(3 z-z\left(q+\frac{\Delta_{2}-\Delta_{1}}{4}\right)+1\right)}-\frac{1}{2}\left(\frac{1}{\Delta_{1}} \ln \frac{q}{q-\Delta_{1}}-\frac{1}{\Delta_{2}} \ln \frac{q}{q+\Delta_{2}}\right) \frac{B^{2}\left(C_{b}+C_{h}\right)}{\left(3 z-z\left(q+\frac{\Delta_{2}-\Delta_{1}}{4}\right)+1\right)}
\end{aligned}
$$

The first and the second derivatives of $\pi(\tilde{z}, B)$ with respect to $Z$ and $B$ are obtained to find the maximum profit in fuzzy sense. The following are the expressions for both the derivatives. $\frac{\sim \tilde{d \pi, B)}}{d z}=\left(\frac{1}{\left(3 z-z\left(q+\frac{\Delta_{2}-\Delta_{1}}{4}\right)+1\right)^{2}}\right)$

$\left[\begin{array}{l}\frac{1}{2}\left(\frac{1}{\Delta_{1}} \ln \frac{q}{q-\Delta_{1}}-\frac{1}{\Delta_{2}} \ln \frac{q}{q+\Delta_{2}}\right)\left(2 D S_{g}-2 D C_{p}-2 D C_{s}+6 D C_{k}-2 D C_{k}\left(q+\frac{\Delta_{2}-\Delta_{1}}{4}\right)\right) \\ \left.-\begin{array}{l}\left.6 C_{h} z^{2}-5 C_{h} z^{2}\left(q+\frac{\Delta_{2}-\Delta_{1}}{4}\right)+C_{h} z^{2}\left(q+\frac{\Delta_{2}-\Delta_{1}}{4}\right)^{2}\right) \\ +4 C_{h} z-2 C_{h} z\left(q+\frac{\Delta_{2}-\Delta_{1}}{4}\right)-2 B C_{h} \\ +\frac{1}{2}\left(\frac{1}{\Delta_{1}} \ln \frac{q}{q-\Delta_{1}}-\frac{1}{\Delta_{2}} \ln \frac{q}{q+\Delta_{2}}\right)\left(3 B^{2} C_{b}-B^{2} C_{b}\left(q+\frac{\Delta_{2}-\Delta_{1}}{4}\right)+3 B^{2} C_{h}-B^{2} C_{h}\left(q+\frac{\Delta_{2}-\Delta_{1}}{4}\right)\right)\end{array}\right]\end{array}\right.$

$\frac{\partial \pi \tilde{(z, B)}}{\partial B}=\frac{2 C_{h} z}{\left(3 z-z\left(q+\frac{\Delta_{2}-\Delta_{1}}{4}\right)+1\right)}-\frac{1}{2}\left(\frac{1}{\Delta_{1}} \ln \frac{q}{q-\Delta_{1}}-\frac{1}{\Delta_{2}} \ln \frac{q}{q+\Delta_{2}}\right) \frac{2 B\left(C_{b}+C_{h}\right)}{\left(3 z-z\left(q+\frac{\Delta_{2}-\Delta_{1}}{4}\right)+1\right)}$

By setting (2.2.5(a)) and (2.2.5(b)) equal to zero, we obtain:

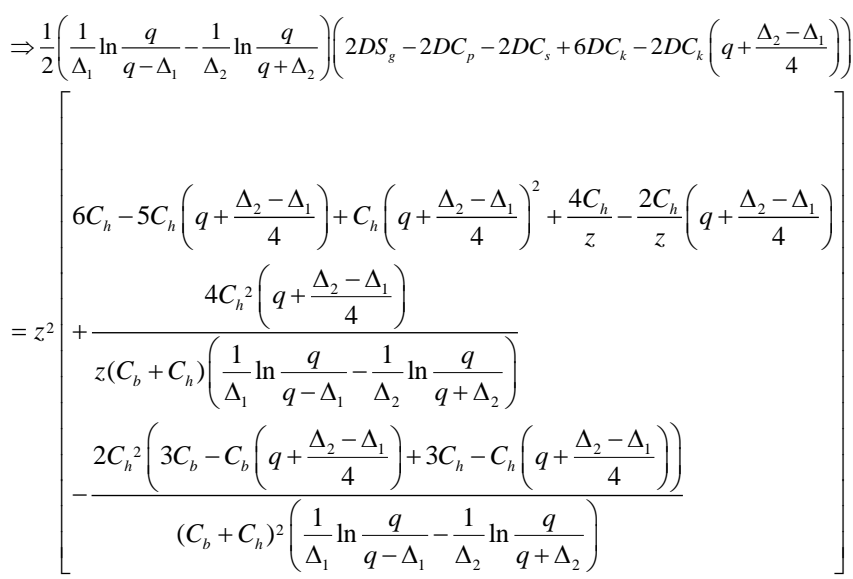

For large value of $z, \frac{1}{z} \rightarrow 0$

$$
z^{*}=\sqrt{\frac{\frac{1}{2}\left(\frac{1}{\Delta_{1}} \ln \frac{q}{q-\Delta_{1}}-\frac{1}{\Delta_{2}} \ln \frac{q}{q+\Delta_{2}}\right)\left(2 D S_{g}-2 D C_{p}-2 D C_{s}+6 D C_{k}-2 D C_{k}\left(q+\frac{\Delta_{2}-\Delta_{1}}{4}\right)\right)}{6 C_{h}-5 C_{h}\left(q+\frac{\Delta_{2}-\Delta_{1}}{4}\right)+C_{h}\left(q+\frac{\Delta_{2}-\Delta_{1}}{4}\right)^{2}}}
$$

$B^{*}=\left(\frac{2 C_{h} z}{\left(C_{b}+C_{h}\right)\left(\frac{1}{\Delta_{1}} \ln \frac{q}{q-\Delta_{1}}-\frac{1}{\Delta_{2}} \ln \frac{q}{q+\Delta_{2}}\right)}\right)$

$I M=z-B$

In other hand by replacing (2.2.6) and (2.2.7) in equation (2.2.8) we have:

$I M^{*}=\frac{z^{*}\left(C_{b}+C_{h} E[p]\right)}{C_{b}+C_{h}}$ 
In order to examine the second-order sufficient conditions (SOSC) for maximum value, we first obtain the Hessian matrix $\mathrm{H}$ which is given as follows:

$H=\left[\begin{array}{cc}\frac{\partial^{2} \pi(z, B)}{\partial z^{2}} & \frac{\partial^{2} \pi(z, B)}{\partial z \partial B} \\ \underset{\sim}{\sim} & \sim \\ \frac{\partial^{2} \pi(z, B)}{\partial B \partial z} & \frac{\partial^{2} \pi(z, B)}{\partial B^{2}}\end{array}\right]$

$\frac{\partial^{2} \pi(z, B)}{\partial z^{2}}==\left(\frac{-1}{\left(3 z-z\left(q+\frac{\Delta_{2}-\Delta_{1}}{4}\right)+1\right)^{3}}\right)$

$\left[\begin{array}{l}\left(3-\left(q+\frac{\Delta_{2}-\Delta_{1}}{4}\right)\right)\left(\frac{1}{\Delta_{1}} \ln \frac{q}{q-\Delta_{1}}-\frac{1}{\Delta_{2}} \ln \frac{q}{q+\Delta_{2}}\right) \\ \left(\begin{array}{l}2 D S_{g}-2 D C_{p}-2 D C_{s}+6 D C_{k} \\ -2 D C_{k}\left(q+\frac{\Delta_{2}-\Delta_{1}}{4}\right)\end{array}\right) \\ +3 B^{2} C_{b}-B^{2} C_{b}\left(q+\frac{\Delta_{2}-\Delta_{1}}{4}\right) 3 B^{2} C_{h} \\ -B^{2} C_{h}\left(q+\frac{\Delta_{2}-\Delta_{1}}{4}\right)+4 C_{h}-2 C_{h}\left(q+\frac{\Delta_{2}-\Delta_{1}}{4}\right)\end{array}\right]$

$\frac{\partial^{2} \pi(z, B)}{\partial z \partial B}=\frac{1}{\left(3 z-z\left(q+\frac{\Delta_{2}-\Delta_{1}}{4}\right)+1\right)^{2}}$

$\left[2 C_{h}+\left(\frac{1}{\Delta_{1}} \ln \frac{q}{q-\Delta_{1}}-\frac{1}{\Delta_{2}} \ln \frac{q}{q+\Delta_{2}}\right) B\left(C_{b}+C_{h}\right)\left(3-\left(q+\frac{\Delta_{2}-\Delta_{1}}{4}\right)\right)\right]$

$$
\frac{\partial^{2} \pi(z, B)}{\partial B \partial z}=\frac{1}{\left(3 z-z\left(q+\frac{\Delta_{2}-\Delta_{1}}{4}\right)+1\right)^{2}}
$$

$\left[2 C_{h}+\left(\frac{1}{\Delta_{1}} \ln \frac{q}{q-\Delta_{1}}-\frac{1}{\Delta_{2}} \ln \frac{q}{q+\Delta_{2}}\right) B\left(C_{b}+C_{h}\right)\left(3-\left(q+\frac{\Delta_{2}-\Delta_{1}}{4}\right)\right)\right]$

$\frac{\partial^{2} \pi \tilde{(z, B)}}{\partial B^{2}}=-\left(\frac{1}{\Delta_{1}} \ln \frac{q}{q-\Delta_{1}}-\frac{1}{\Delta_{2}} \ln \frac{q}{q+\Delta_{2}}\right) \frac{\left(C_{b}+C_{h}\right)}{\left(3 z-z\left(q+\frac{\Delta_{2}-\Delta_{1}}{4}\right)+1\right)}$

Taking $\Delta_{1}=\Delta_{2}=\Delta$ in Eq. (2.2.6), we get the following equation.

$$
z^{*}=\sqrt{\frac{\frac{1}{2}\left(\frac{1}{\Delta} \ln \frac{q}{q-\Delta}-\frac{1}{\Delta} \ln \frac{q}{q+\Delta}\right)\left(2 D S_{g}-2 D C_{p}-2 D C_{s}+6 D C_{k}-2 D C_{k} q\right)}{6 C_{h}-5 C_{h} q+C_{h} q^{2}-\frac{2 C_{h}\left(3 C_{b}-C_{b} q+3 C_{h}-C_{h} q\right)}{\left(C_{b}+C_{h}\right)^{2}\left(\frac{1}{\Delta} \ln \frac{q}{q-\Delta}-\frac{1}{\Delta} \ln \frac{q}{q+\Delta}\right)}}}
$$

$B^{*}=\left(\frac{2 C_{h} z}{\left(C_{b}+C_{h}\right)\left(\frac{1}{\Delta} \ln \frac{q}{q-\Delta}-\frac{1}{\Delta} \ln \frac{q}{q+\Delta}\right)}\right)$

When $\Delta \rightarrow 0$ by applying L'Hospital's Rule $\frac{1}{\Delta} \ln \left(\frac{q+\Delta}{q-\Delta}\right)=\frac{2}{q}$

$z=\sqrt{\frac{\frac{1}{q}\left(2 D S_{g}-2 D C_{p}-2 D C_{s}+6 D C_{k}-2 D C_{k} q\right)}{6 C_{h}-5 C_{h} q+C_{h} q^{2}-\frac{C_{h}^{2}(3-q)}{\left(C_{b}+C_{h}\right) \frac{1}{q}}}}$

$B=\left(\frac{C_{h} z q}{\left(C_{b}+C_{h}\right)}\right)$

When $q=1$ and $C_{s}+C_{p}=S_{g}$ in Eq. (2.2.17), $z$ reduces to the traditional EOQ backorder formula for the lot size i.e.,

$z=\sqrt{\frac{2 C_{k} D}{C_{h}}\left(\frac{C_{b}+C_{h}}{C_{b}}\right)}$

\section{NUMERICAL RESULTS}

To illustrate the proposed models, we have considered the following examples:

\section{Example-3.1: (Crisp Model)}

$D=50,000$ units/year, $C_{k}=100 /$ cycle, $C_{h}=\$ 5 /$ unit/year $w$ $=1$ unit $/ \mathrm{min}, C_{s}=0.5 /$ unit, $C_{p}=25 /$ unit, $C_{b}=\$ 20 /$ unit, $S_{g}=$ \$50/unit,

[We assume the operation of the inventory model operates 8 hours a day, for 365 days a year, so the annual inspection rate is $w=175200$ units/year]

Also, we assume the defective rate $p$ to be uniformly distributed with p.d.f given as follows:

$f(p)= \begin{cases}25, & 0 \leq \mathrm{p} \leq 0.05 \\ 0 & \text { otherwise }\end{cases}$

The expected values present in the model are given as follows:

$$
E[p]=\int_{a}^{b} p f(p) d p=\int_{0}^{0.04} 25 p d p=0.02
$$

and

$$
\begin{aligned}
E[1 /(1-p)] & =\int_{a}^{b}(1 /(1-p)) f(p) d p \\
& =25 \int_{0}^{0.04}(1 /(1-p)) d p=1.02055
\end{aligned}
$$


The optimal value of $z^{*}$ and $B$ that optimizes the expected total profit per unit time is given by $z^{*}=1648.88, Q^{*}=323.182$ and $I M^{*}=1325.698$

\section{Example-3.2: Fuzzy Model}

$D=50000$ units/year, $C_{k}=\$ 100 /$ cycle, $C_{h}=\$ 5=$ unit $/$ year, $w=1$ unit $/ \min$ (equivalently, $w=175200$ units/year), $C_{s}=\$ 0.5 /$ unit, $C_{p}=\$ 25 /$ unit, $S_{g}=\$ 50 /$ unit and we consider that the defective rate is around $p=0.02$ (i.e., the goodquality rate is around $q=1-p=0.98$ ).

$p=0.02, q=1-p=0.98, \Delta_{1}=0.00550, \Delta_{2}=0.00455$

The expected total profit per unit time $\pi(z, B)$ and the optimal lot size $z$ are evaluated to be 1230990/year and 1666.83 units respectively.

$$
Q^{*}=326.528 \text { and } I M^{*}=1340.302
$$

\section{IIL}

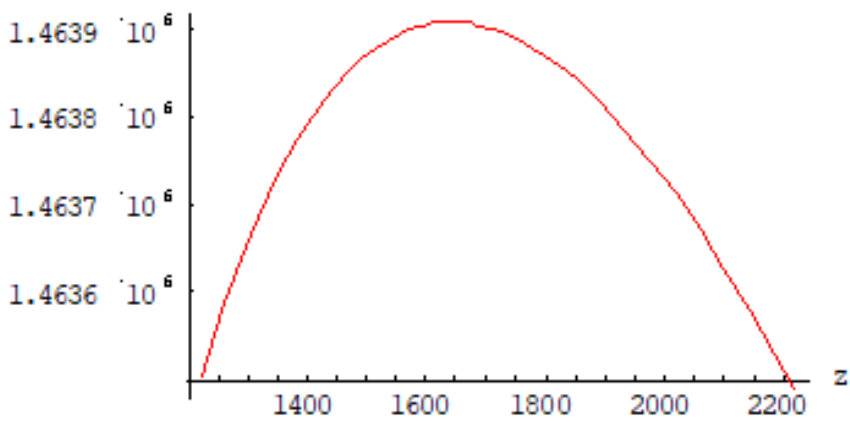

Figure 2. $\pi(z, B)$ vs $Z$

Table 2. For fuzzy model

\begin{tabular}{|c|c|c|c|c|c|}
\hline Parameters & $\begin{array}{l}\text { Percentage } \\
\text { change }\end{array}$ & $z$ & $\bar{B}$ & $\pi(z, B)$ & $\begin{array}{c}\text { \%change in } \\
\sim(z, B)\end{array}$ \\
\hline \multirow[t]{4}{*}{$D$} & -25 & 1863.07 & 365.07 & 1539640 & $25.07 \%$ \\
\hline & +15 & 1786.99 & 350.162 & 1416170 & $15.04 \%$ \\
\hline & -15 & 1536.32 & 301.043 & 1045850 & $-15.04 \%$ \\
\hline & -25 & 1443.12 & 282.78 & 922456 & $-25.06 \%$ \\
\hline \multirow[t]{4}{*}{$S_{t}$} & -25 & 1711.74 & 335.417 & 1862330 & $51.29 \%$ \\
\hline & +15 & 1693.74 & 331.89 & 1609800 & $30.77 \%$ \\
\hline & -15 & 1638.56 & 321.077 & 852190 & $-30.77 \%$ \\
\hline & -25 & 1619.75 & 317.391 & 599656 & $-51.29 \%$ \\
\hline \multirow[t]{4}{*}{$C_{i}$} & +25 & 1842.8 & 361.098 & 1230270 & $-0.06 \%$ \\
\hline & +15 & 1774.34 & 347.683 & 1230550 & $-0.04 \%$ \\
\hline & -15 & 1550.91 & 303.902 & 1231460 & $0.04 \%$ \\
\hline & -25 & 1468.91 & 287.834 & 1231800 & $0.07 \%$ \\
\hline \multirow[t]{4}{*}{$C_{r}$} & +25 & 1643.23 & 321.992 & 915324 & $-25.64 \%$ \\
\hline & +15 & 1652.53 & 323.815 & 1041590 & $-15.39 \%$ \\
\hline & -15 & 1680.11 & 329.219 & 1420390 & $15.39 \%$ \\
\hline & -25 & 1689.21 & 331.002 & 1546660 & $25.64 \%$ \\
\hline \multirow[t]{4}{*}{$C_{s}$} & +25 & 1665.92 & 326.438 & 1224680 & $-0.51 \%$ \\
\hline & +15 & 1666.1 & 326.474 & 1227200 & $-0.31 \%$ \\
\hline & -15 & 1666.65 & 326.581 & 1234780 & $0.31 \%$ \\
\hline & -25 & 1666.84 & 326.619 & 1237310 & $0.51 \%$ \\
\hline \multirow[t]{4}{*}{$\bar{q}$} & +25 & 1872.01 & 458.551 & 1121240 & $-8.29 \%$ \\
\hline & +15 & 1760.12 & 396.645 & 1154680 & $-6.2 \%$ \\
\hline & -15 & 1634.42 & 272.311 & 1349970 & $9.67 \%$ \\
\hline & -25 & 1642.36 & 241.345 & 1463910 & $18.92 \%$ \\
\hline \multirow[t]{4}{*}{$C_{h}$} & -25 & 1525.39 & 355.835 & 1230360 & $-0.05 \%$ \\
\hline & +15 & 1575.88 & 344.771 & 1230600 & $-0.03 \%$ \\
\hline & -15 & 1781.43 & 305.888 & 1231430 & $0.04 \%$ \\
\hline & -25 & 1877.75 & 290.484 & 1231760 & $0.06 \%$ \\
\hline \multirow[t]{4}{*}{$b$} & +25 & 1634.31 & 266.87 & 1230860 & $-0.01 \%$ \\
\hline & +15 & 1645.55 & 287.899 & 1230910 & $-0.01 \%$ \\
\hline & -15 & 1694.06 & 377.219 & 1231100 & $0.01 \%$ (T) \\
\hline & -25 & 1718.22 & 420.858 & 1231200 & $0.02 \%$ \\
\hline
\end{tabular}

\section{OBSERVATIONS}

From the above table we observed the following:

i. To make the model more realistic it has been assumed the rate of defective to be a fuzzy number. Under this circumstance, it is observed that when there is an increase in the percentage of defectives, the optimum lot size increases both for increase or decrease in the percentage of defectives, but the total profit is becoming smaller in amount than the actual $\pi(z, B)$, and vise-a-versa.

ii. When the parameters $D$ and $S_{g}$ are increased by $25 \%$ and $15 \%$, there is an increase in both $z$ and $\pi(z, B)$. Similarly, when $D$ and $S_{g}$ are decreased by $15 \%$ and $25 \%$, there is a decrease in both $z$ and $\pi(z, B)$.

iii. When the parameter $C_{k}$ is increased by $25 \%$ and $15 \%$, there is an increase in the value of $z$ and decrease in the value of $\pi(z, B)$. Similarly, when $C_{k}$ is decreased by $15 \%$ and $25 \%$, there is a decrease in the value of $z$ and increase in the value of $\pi(\tilde{z}, B)$. When the parameters $C_{p}, C_{s}$ and $C_{h}$ are increased by $25 \%$ and $15 \%$, there is decrease in both $z$ and $\pi(z, B)$. Similarly, when $C_{p}, C_{s}$ and $C_{h}$ are decreased by $15 \%$ and $25 \%$, there is an increase in both $z$ and $\pi(z, B)$.

\section{CONCLUSION}

In this paper, we have discussed a fuzzy EOQ inventory model for imperfect items with shortages. In both crisp and fuzzy type, allowable proportionate discounts have been introduced depending on the number of defective items present in each lot. It is observed that the rate of discount increases when the number of defectives decrease. A $100 \%$ screening of the received item are done in case of both crisp as well as fuzzy model and the defective items are sold as a single batch with the estimated proportionate discount. It is observed that both the models reduce to the traditional backorder EOQ model formulae and the profits are found to be more or less same as that of the profits obtained in case of the Rezaei [18] and Chang [1]. For each model, it is found that the lot size of the models increases as the $\%$ of defectives increases. The optimum results with regard to the lot size and the total profit of fuzzy models are defuzzified by Signed distance method. Finally, we would like to point out on the results obtained out of the sensitivity analysis conducted with regard to the changes in percentages made for the different parameters involved in the fuzzy models. The changes made for the parameters, giving us the lot size and total profit to be mostly symmetric in nature. Also, the graphs are plotted for the total profit versus lot size and the difference of the profits of the (crisp/fuzzy) model versus the lot size.

\section{REFERENCES}

[1] Chang HC. (2004). An implication of fuzzy sets theory to the EOQ model with imperfect quality items. 
Computers and Operations Research 31(12): 2079-2092. https://doi.org/10.1016/S0305-0548(03)00166-7

[2] Chang HC, Ho CH. (2010). Exact closed-form solutions for optimal inventory model for items with imperfect quality and shortage backordering. Omega 38(3-4): 233-237. https://doi.org/10.1016/j.omega.2009.09.006

[3] Chen SH, Chang SM. (2008). Optimization of fuzzy production inventory model with unrepairable defective products. International Journal of Production Economics 113(2): 887-894. https://doi.org/10.1016/j.ijpe.2007.11.004

[4] De PK, Rawat A. (2011). A fuzzy inventory model without shortages using triangular fuzzy number. Fuzzy Information and Engineering 3: 59-68. https://doi.org/10.1007/s12543-011-0066-9

[5] Eroglu A, Ozdemir, A. (2007). An economic order quantity model with defective items and shortages. International Journal of Production Economics 106: 544-49. https://doi.org/10.1016/j.ijpe.2006.06.015

[6] Goyal SK, Cardenas-Barron LE. (2002). 'Note on: Economic production quantity model for items with imperfect quality- a practical approach. International Journal of Production Economics 77: 85-87. https://doi.org/10.1016/S0925-5273(01)00203-1.

[7] Harris FW. (1913). Operations and costs (Factory Management Series). A.W. Shaw Co, Chicago, pp. 1852.

[8] Hsu JT, Hsu LF. (2012). A note on: 'Optimal inventory model for items with imperfect quality and shortage backordering. International Journal of Industrial Engineering Computations 3(5): 939-948. https://doi.org/10.5267/j.ijiec.2012.05.007.

[9] Jagadeeswari J, Chenniappan PK. (2014). An order level inventory model for deteriorating items with time - quadratic demand and partial backlogging. Journal of Business and Management Sciences. 2(3): 79-82. https://doi.org/10.12691/jbms-2-3-3

[10] Khana A, Gautam P, Jaggi CK. (2017). Inventory Modelling for deteriorating imperfect quality items with selling price dependent demand and shortage backordering under credit financing. International Journal of Mathematical, Engineering and Management Sciences 2(2): 110-124.

[11] Kumar RS, Goswami A. (2013). Fuzzy stochastic EOQ inventory model for items with imperfect quality and shortages are backlogged. AMO - Advanced Modeling and Optimization 15(2): 261-279.

[12] Lee HL, Rossenblatt MJ. (1987). Simultaneously determination of production cycles and inspection schedules in a production system. Management Science 33: 1125-1137.

[13] Lee HM, Yao JS. (1998). Economic production quantity for fuzzy demand quantity and fuzzy production quantity. European Journal of Operational Research 109(1): 203-211. https://doi.org/10.1016/S03772217(97)00200-2

[14] Maddah B, Jaber MY. (2008). Economic order quantity for items with imperfect quality: revisited. International Journal of Production Economics 112(2): 808-815. https://doi.org/10.1016/j.ijpe.2007.07.003

[15] Patro R, Acharya M, Nayak MM, Patnaik S. (2017). A fuzzy inventory model with time dependent Weibull deterioration, quadratic demand and partial backlogging. International Journal Management and Decision
Making 16(3):

243-279.

https://doi.org/10.1504/IJMDM.2017.085636

[16] Patro R, Acharya M, Nayak MM, Patnaik S. (2017). A fuzzy imperfect quality inventory model with proportionate discount under learning effect. International Journal of Intelligent Enterprise 4(4): 303327.

[17] Porteus EL. (1986). Optimal lot sizing, process quality improvement and setup cost reduction. Operations Research 34: 137-144. https://doi.org/10.1287/opre.34.1.137

[18] Rezaei J. (2005). Economic order quantity model with backorder for imperfect quality items. Proceeding of IEEE International Engineering Management Conference, pp. 466-470.

[19] Rossenblatt MJ, Lee HL. (1986). Economic production cycles with imperfect production processes. IIE Transactions 18: 48-55 https://doi.org/10.1080/07408178608975329

[20] Salameh MK, Jaber MY. (2000). Economic production quantity model for items with imperfect quality. International Journal of Production Economics Vol. 64: 59-64. https://doi.org/10.1016/S0925-5273(99)00044-4

[21] Sujatha J, Parvathi P. (2015). Fuzzy EOQ model for deteriorating items with weibull demand and partial backlogging under trade credit. International Journal of Informative and Futuristic Research 3(2): 526-537.

[22] Wahab MIM, Jaber MY. (2010). Economic order quantity model for items with imperfect quality, different holding costs, and learning effects: A note. Computers and Industrial Engineering 58(1): 186-190. https://doi.org/10.1016/j.ijpe.2010.01.023.

[23] Wee HW, Yu J, Chen MC. (2007). Optimal inventory model for items with imperfect quality and shortage backordering. Omega 35(1): 7-11, 13 https://doi.org/10.5267/j.ijiec.2012.05.007

[24] Zadeh LA, Bellman RE. (1970). Decision making in a fuzzy environment. Management Science 17: 140-164. https://doi.org/10.1287/mnsc.17.4.B141

\section{NOTATIONS AND ASSUMPTIONS}

\section{Notations}

\section{Crisp Notations}

$z \quad$ size of the ordering quantity

$C_{p} \quad$ unit cost of variable

$C_{k} \quad$ ordering cost

$C_{h} \quad$ holding cost

$p \quad \%$ of defective items in $z$

$f(p)$ p.d.f. of $p$

$S_{g} \quad$ selling price of unit wise good quality item

$w \quad$ rate ofscreening

$C_{s} \quad$ unit wise screening cost

$T \quad$ length of one cycle

$C_{b} \quad$ unit wise shortage cost

$Q \quad$ expected shortage level 


\section{CrispAssumptions}

\section{Fuzzy Notations}

1. constant demand rate.

\section{$\tilde{\bar{q}} \quad$ fuzzy defective rate}

$\pi(z, B) \quad$ unit time wise total profit in fuzzy sense

$d\left(\tilde{\bar{q}}, \tilde{0}_{1}\right) \quad$ signed distance of fuzzy number $\tilde{\bar{q}}$ to $\tilde{0}_{1}$

$d\left(\frac{1}{\tilde{\bar{q}}}, \tilde{0}_{1}\right) \quad$ signed distance of fuzzy number $\frac{1}{\tilde{\bar{q}}}$ to $\tilde{0}_{1}$

2. instantaneous delivery of lot size

3. lead time is zero.

4. shortages are permitted.

5. imperfect quality items do exist in the lot.

6. $100 \%$ screening process is conducted in each lot.

\section{Fuzzy Assumptions}

- Fuzzy defective rate

- Triangular fuzzy number is considered for defective rate

- Signed distance method is used for defuzzification. 